\title{
Age dependent changes of cell update markers and endothelial activity related to the involutionary transformations of the skin system.
}

\author{
Manturova NE ${ }^{1,2}$, Kononov $\mathrm{AV}^{3}$, Gorodilov $\mathrm{RV}^{4}$, Stupin $\mathrm{VA}^{1,2}$, Litvitsky $\mathrm{PF}^{4}$, Silina $\mathrm{EV}^{4 *}$ \\ ${ }^{1}$ Institute of Plastic Surgery and Cosmetology; Olkhovskaya St, 27, Moscow, Russia \\ ${ }^{2}$ Pirogov Russian National Research Medical University (RNRMU), Ostrovityanova St. 1, Moscow, Russia \\ ${ }^{3}$ Omsk State Medical University; Lenina St, 12, Omsk, Russia \\ ${ }^{4}$ Sechenov First Moscow State Medical University (Sechenov University); Trubetskaya St, 8, Moscow, Russia
}

\begin{abstract}
Aim: The main aim of the study is to examine the collagen-elastic skeleton of the skin and markers of cellular renewal in the epidermis during aging, as well as age-related changes in the expression of endothelial markers of human skin.

Materials and methods: The material of the study was the skin fragments taken during the first 4-12 h after death of 20 women under the age of $35 \mathrm{y}$ and of 24 women over $50 \mathrm{y}$. All women were of the Eurasian race. Evaluation of collagen and elastic fibers was carried out by means of coloring according to Weigert-Van Gieson. Level of expression of Ki-67 and p53 by epidermocyte was determined by immunohistochemical methods, and also the expression of CD31 and ICAM-1 receptors on endothelial cells of skin fragments obtained by autopsy of corpses were evaluated.

Results: With aging, against an increase in the number of skin elastic fibers, the amount of collagen fibers reduced. At the same time, the amount of decorin in the extracellular matrix decreased. In older age groups, the Ki-67 label index decreased, while the expression level of p53 in the epidermis was low in young people.

Discussion: A significant reduction in the number of capillaries of the papillary layer and the number of CD31-endotheliocytes in the older age group was found, while the level of ICAM-1 expression in the study groups did not change.
\end{abstract}

Keywords: Aging, Involution, Skin, Dermis, Epidermis, Collagen, Elastin, Ki-67, p53, CD31.

Accepted on November 5, 2018

\section{Introduction}

The increase in the duration and quality of life of developed countries population is accompanied by a progressive increase in the number of plastic surgeries on the face. This made it necessary to search for precise information about the mechanisms and predictors of aging. Age changes in the skin subtly reflect the complex processes of organism aging and allow determining the biological age of the person with great probability, as well as the presence of various forms of pathology in him. The state of the skin system depends on the influence of environmental and hereditary factors and the way of life. One of the most important mechanisms of skin aging is the gradual loss of function or degeneration that occurs at the cellular level. Primary cultured cells undergo replicative aging, which is characterized by shortening telomeres, genomic damage, epigemous damage and activation of tumor processes $[1]$.
Currently, the issues of skin condition in clinical practice are given great importance, and the number of studies evaluating age-related changes in the skin has a persistent tendency to increase. Biomarkers of involute skin processes [2] and indicators of skin biomechanics at different stages of aging [3] are actively studied. A very important marker of aging today is reactive oxygen species (ROS), which are mainly associated with oxidative cellular metabolism. ROS play an important role both in chronological aging and in the visual manifestation of facial skin aging $[4,5]$. Therefore, an adequate and timely assessment of these markers is very important in the practice of plastic surgeons and cosmetologists. At a macroscopic level, the aging of the dermis is mechanically expressed in an increase in stiffness, a decrease in moisture and the ability to repel [6]. At the microscopic level, the complex multiparametric aging process ultimately leads to a decrease in the collagen and elastin content due to an imbalance between the synthesis and degradation of matrix proteins by matrix metalloproteinases, increased collagen crosslinking, 
deterioration of proteoglycans followed by water loss [7-9]. However, the relationship between these structural modifications and mechanical changes is still an unresolved issue. In order to identify and describe the regularities of the flow of the above-described processes, a comprehensive study of the state of human skin fragments has been conducted in this study.

The aim of the study was to study the collagen-elastic skeleton of the skin and markers of cellular renewal in the epidermis during aging, as well as age-related changes in the expression of endothelial markers in skin vessels in people of different ages.

To achieve this goal, the following main tasks were set.

1. To conduct a comparative histological and histochemical study of the skin condition with the identification and description of significant signs of differences in the histoarchitectonics of the collagen-elastic dermal framework in individuals younger than $35 \mathrm{y}$ and over $50 \mathrm{y}$;

2. To determine the features of $\mathrm{Ki}-67$ expression (the marker of tumor cell proliferative activity) [10-12], the p53 protein (transcription factor regulating the cell cycle and performing the function of suppressor of blastomogenesis) [12-14] in the individuals of the specified age periods;

3. To identify patterns of glycoprotein CD31 (participating in angiogenesis processes and its prognostic marker) $[15,16]$ and ICAM-1-positive cells (carrying a molecule of cell adhesion on the membranes of leukocytes and endothelial cells, the expression of which significantly increases under the influence of cytokines, especially interleukin-1 (IL-1) and tumor necrosis factor-alpha tumor $(\mathrm{TNF}-\alpha))[17,18]$.

\section{Material and Methods}

Skin fragments obtained during an autopsy performed during the first 4-12 $\mathrm{h}$ after the death was detected in 20 young women aged $18 \mathrm{y}$ to $35 \mathrm{y}(29.1 \pm 3.1 \mathrm{y}$-group $1 \mathrm{~A})$ and 24 women older $50 \mathrm{y}$ (from $51 \mathrm{y}$ to $80 \mathrm{y}, 59.7 \pm 8.3 \mathrm{y}-2^{\text {nd }}$ group B). All women were of the Eurasian race. The causes of death in this study were not analysed. Skin patches measuring $5 \times 3$ $\times 3 \mathrm{~mm}$ were excised in the chin area from the sections adjacent to the median incision. Fragments of the skin were fixed in $10 \%$ buffered formalin. The treatment and filling into paraffin was carried out according to a standard procedure. From the blocks obtained, slices $4 \mu \mathrm{m}$ thick were prepared, which for stunning study were stained with hematoxylin and eosin. To identify the elastic and collagen skeleton of the skin, the corresponding fibers were differentiated by the coloring of Weigert-Van Gison. For morphometry, a square grid measuring $10 \times 10\left(10^{5} \times 10^{5} \mu \mathrm{m}\right)$ with 121 points of interest was used. After the mesh was overlaid on the image obtained at a 1000fold magnification of the microscope, the number of intersection points with collagen and elastic fibers was counted. The results of the study of 10 random fields of vision were summarized and the volume fraction of the structures of interest was calculated. The cell renewal process was evaluated immunohistochemically, according to the expression level of Ki-67 molecules (proliferation marker) using mouse DakoCytomation antibodies, clone MIB-1. Expression of the p53 molecule (regulatory cell cycle), as well as CD31 and ICAM-1 adhesion molecules, was tested using murine Novocastr-derived antibodies, clone DO-7. Counting the number of positive keratinocytes in 5 random fields of vision, the index of the label was calculated per 100 cells of the epidermis. Estimation of the expression degree of the decorin protein was carried out by immunohistochemical reaction with murine 9XX clone antibodies (Santa Cruz Biotechnology).

Statistical analysis of the results was carried out using the software SPSS 10. The results of the statistical analysis are presented as the mean value (Median; Me), and lower $(25 \%)$ and upper (75\%) quartiles (Q25/Q75). A comparative analysis was performed using the Mann-Whitney (U) test. Differences were considered significant with an error level of $p<0.05$.

\section{Results and Discussion}

Histological characteristics of the individual's skin in group A were characterized by a normal structure of all skin levels. Histochemical examination of the papillary layer region and the upper third of the dermis revealed ramified elastic fibers that braided bundles of collagen. The bulk density of the elastic fibers averaged 6 for the interquartile interval (Q25/Q75)-6/7. The bulk density of collagen fibers, unlike the elastic ones, was significantly higher $(p<0.05)$ and averaged 43 for the interquartile range of $42 / 45$. Histological examination of the older age group skin (B) revealed a decrease in the height of the papillae of the dermis and a thinning of the epidermis. This was due to a decrease in the number of its layers. The border between the epidermis and the dermis lost the tortuosity characteristic of the young. Attention was drawn to the decrease in the number of collagen and the increase in elastic fibers. The latter looked thick, homogeneous, and coarse. The volume fraction of elastic fibers was on average $17 \%$ higher than for women of group A, averaging $7(\mathrm{Q} 25=7$, Q75=8), and the bulk density of collagen fibers, on the contrary, decreased by an average of $12 \%(\mathrm{Me}=38, \mathrm{Q} 25=37, \mathrm{Q} 75=41, \mathrm{p}<0.05)$.

Results of the immunohistochemical study showed that the increase in signs of skin involution with a decrease in the thickness of its epidermis in women after $50 \mathrm{y}$ correlated with a significant decrease (by 1.5 times) in the number of functionally active cells of the dermis. Thus, in young women, Ki-67 expression was detected in the nuclei of cells predominantly in the basal layer, and in adults over $50 \mathrm{y}$, positive Ki-67 keratinocytes were located in both the basal and suprabasal layers. The average number of $\mathrm{Ki}-67$ positive keratinocytes in group A was $8.2(\mathrm{Q} 25=7.5$, Q75=9.6), in group B-5.4 (Q25=4.7, Q75=5.9) for 100 cells $(\mathrm{p}<0.05)$.

Expression of p53 in young women was noted in the keratinocyte nuclei of the suprabasal layer and was weak in intensity. On average, the index of p53 in group A was 2.6 (Q25=1.7, Q75=3.8) per 100 cells. Significantly, the highest expression for $\mathrm{p} 53$ was in the papillary and dermal layers of 
the dermis (tested according to the level of the decore-a complex low-molecular protein from the group of proteoglycans necessary for the process of collagen assembly). The most intensive staining was associated with the presence of thick collagen fibers. Immunohistochemical examination of the women skin of group B showed an increase in the area of the compartment of p53 positive epidermocytes, their number increased on average to $4.2(\mathrm{Q} 25=3.0, \mathrm{Q} 75=5.6)$ for 100 cells, in 1.62 times $(\mathrm{p}<0.05)$. The intensity of coloring of the decor in group B was lower, and the label was located around the collagen fibers in all compartments of the dermis. At the same time, the greatest increase was observed around the thin collagen fibers of the papillary layer.

When staining sections with hematoxylin and eosin, the thinning of the epidermis (especially the papillary layer of the dermis), substantially dependent on age, and smoothing of the basal membrane was noted. At the same time, the intensity of coloring of the fibrillar structures decreased. The architecture of the capillaries of the papillary layer remained unchanged, but the number of capillaries decreased statistically significant, on average by a quarter from $58(50.3 / 61.5)$ in $1 \mathrm{~cm}^{2}$ in group A to $44(39.1 / 51.3)$ per $\mathrm{cm}^{2}$ in group $\mathrm{B}(\mathrm{U}=20.0, \mathrm{p}<0.05)$.

In the immunohistochemical study of women skin samples of group A, CD31 molecules were visualized on membranes of endothelial cells lining the vessels mainly in the papillary dermis. Their density was an average of 51.5 (45.6/55.6 per 1 $\mathrm{cm}^{2}$ ). In the reticular layer of the dermis, endotheliocytes did not express this marker. The presence of ICAM-1 was noted on endothelial cells in the papillary layer $\left(\mathrm{Me}=32.5\right.$ per $\mathrm{cm}^{2}, \mathrm{Q} 25 /$ $\mathrm{Q} 75=29.0 / 36.8$ per $\mathrm{cm}^{2}$ ). In women of group $\mathrm{B}$, the CD31 receptor was found on the cytoplasmic membrane of endotheliocytes. The intensity of the label did not differ from group A, but the amount of CD31 molecules decreased significantly, averaging $36.4\left(\mathrm{Q} 25 / \mathrm{Q} 75=32.5 / 47.3\right.$ per $\left.1 \mathrm{~cm}^{2}\right)$, which is $30 \%$ less $(\mathrm{U}=18.0, \mathrm{p}<0.05)$. In separate fields of vision in extravasal spaces, CD31 positive cells with a pronounced label intensity also occurred. The number of ICAM-1 positive endotheliocytes in group $\mathrm{B}$ tended to decrease $\left(\mathrm{Me}=27.5\right.$ per $1 \mathrm{~cm}^{2}, \mathrm{Q} 25 / \mathrm{Q} 75=26.0 / 33.6$ per $\mathrm{cm}^{2}$; $\mathrm{U}=30.5, \mathrm{p}=0.14)$.

\section{Conclusion}

It is shown that with increasing age there are regular and significant changes in the connective tissue compartment of the dermis. On the one hand, they are characterized by a decrease in the amount of collagen fibers, on the other hand, by an increase in the number of elastic fibers. These changes in the histoarchitectonics of the collagen-elastic skeleton of the skin are accompanied by a loss of its elasticity and an increase in the extensibility leading to its flabbiness. These factors determine, in the main, the age-related changes, including the formation of wrinkles. In return, excessive stretching of the skin leads to a progressive reduction of its papillary layer and a loss of tortuosity of the basal membrane. The described age involution processes in the skin are associated with a decrease in the trophic function of the connective tissue. This, in turn, can cause metabolic disorders in the keratinocytes. A significantly low marker of Ki-67 proliferative activity indicates inhibition of keratinocyte repair processes, which causes hypotrophy and hypoplasia of the epithelial layer of the skin. With aging, there is a decrease in the thickness of the epidermis due to a decrease in the number of its layers. This phenomenon, combined with the loss of tortuosity of the epithelial basement membrane in mature women, significantly reduces the degree of skin protection from external pathogenic factors, including the excess of ultraviolet (UV) rays. It is believed that UV irradiation promotes the initiation of mutations in the genome of skin cells under the influence of excess products of free radical lipid peroxidation. The p53 protein is a highly sensitive marker of skin photodamage [8]. The high expression of p53 molecules in keratinocytes of people aged 50-80 years old is shown by activation of mechanisms of DNA damage repair. It can be assumed that additional mutations can lead to disturbances in the apoptosis process up to its suppression. Thus, the obtained data indicate that the involution process is characterized by signs of hypotrophy of all skin components, and epidermis, and papillary layer, which is associated with a decrease in the number of capillaries and perfusion of blood, lymph, and interstitial fluid. Despite the decrease in the volume of the microcirculatory bed in older women, and therefore the threat of developing relative cellular energy deficiency, endotheliocytes are able to remain in the activated state. This can create conditions for initiating the process of neoangiogenesis and reducing the intensity of the extracellular matrix degradation process, which gives hope for the success of developing effective methods of aging skin inhibition.

\section{References}

1. Campisi J. Aging, cellular senescence, and cancer. Annu Rev Physiol 2013; 75: 685-705.

2. Kanaki T, Makrantonaki E, Zouboulis CC. Biomarkers of skin aging. Rev Endocr Metab Disord 2016; 17: 433-442.

3. Lynch B, Bonod-Bidaud C, Ducourthial G, Affagard JS, Bancelin S, Psilodimitrakopoulos S, Ruggiero F, Allain JM, Schanne-Klein MC. How aging impacts skin biomechanics: a multiscale study in mice. Scientific Reports 2017; 7: 13750.

4. Noh EM, Park J, Song HR, Kim JM, Lee M, Song HK, Hong OY, Whang PH, Han MK, Kwon KB, Kim JS, Lee YR. Skin aging-dependent activation of the pi3k signaling pathway via downregulation of PTEN increases intracellular ROS in human dermal fibroblasts. Oxid Med Cell Longev 2016.

5. Silina EV, Manturova NE, Mamedov EV, Smirnova GO. The clinical and laboratory evaluation of the facial skin state after surgical correction. Khirurgiia 2012; 6: 45-49.

6. Phillip JM, Aifuwa I, Walston J, Wirtz D. The mechanobiology of aging. Annu Rev Biomed Eng 2015; 17: 113-141.

7. Fisher GJ, Quan T, Purohit T, Shao Y, Cho MK, He T, Varani J, Kang S, Voorhees JJ. Collagen fragmentation 
promotes oxidative stress and elevates matrix metalloproteinase-1 in fibroblasts in aged human skin. Am J Pathol 2009; 174: 101-114.

8. Naylor EC, Watson RE, Sherratt MJ. Molecular aspects of skin ageing. Maturitas 2011; 69: 249-256.

9. Hwang K, Yi B, Choi KC. Molecular mechanisms and in vivo mouse models of skin aging associated with dermal matrix alterations. Lab Anim Res 2011; 27: 1-8.

10. El-Hawary AK, Yassin E, Khater A, Abdelgaber S. Expression of matrix metalloproteinase-13 and Ki-67 in nonmelanoma skin cancer in xeroderma pigmentosum and non-xeroderma pigmentosum. Am J Dermatopathol 2013; 35: 45-49.

11. Sun $X$, Kaufman PD2. Ki-67: more than a proliferation marker. Chromosoma 2018; 127: 175-186.

12. Silva DC, Gonçalves AK, Cobucci RN, Mendonça RC, Lima $\mathrm{PH}$, Cavalcanti $\mathrm{G}$ Júnior. Immunohistochemical expression of p16, Ki-67 and p53 in cervical lesions-a systematic review. Pathol Res Pract 2017; 213: 723-729.

13. Jeffers JR, Parganas E, Lee Y, Yang C, Wang J, Brennan J, MacLean KH, Han J, Chittenden T, Ihle JN, McKinnon PJ, Cleveland JL, Zambetti GP. Puma is an essential mediator of p53-dependent and -independent apoptotic pathways. Cancer Cell 2004; 4: 321-328.

14. Gritsenko DA, Orlova OA, Linkova NS, Khavinson VK. Transcription factor p53 and skin aging. Adv Gerontol 2017; 30: 10-16.
15. Mahajan KD, Nabar GM, Xue W, Anghelina M, Moldovan NI, Chalmers JJ, Winter JO. Mechanotransduction effects on endothelial cell proliferation via CD31 and VEGFR2: implications for immunomagnetic separation. Biotechnol J 2017; 12.

16. Wu Q, Zhan J, Pu S, Qin L, Li Y, Zhou Z. Influence of aging on the activity of mice Sca-1+CD31-cardiac stem cells. Oncotarget 2017; 8: 29-41.

17. Yang L, Froio RM, Sciuto TE, Dvorak AM, Alon R, Luscinskas FW. ICAM-1 regulates neutrophil adhesion and transcellular migration of TNF-alpha-activated vascular endothelium under flow. Blood 2005; 106: 584-592.

18. Anbarasan C, Bavanilatha M, Latchumanadhas K, Ajit Mullasari S. ICAM-1 molecular mechanism and genome wide SNPs association studies. Indian Heart J 2015; 67: 282-287.

\section{*Correspondence to}

\section{Silina EV}

Sechenov First Moscow State Medical University (Sechenov University)

Trubetskaya

Moscow

Russia 